\section{Welche Vorteile bringt die Thrombolyse?}

Die Lungenembolie (Pulmonary Embolism, PE) ist eine häufige Todesursache und mit einer erhöhten Sterblichkeit bis zu 3 Monate nach dem Ereignis verbunden. Das optimale Vorgehen bei einer PE wird kontrovers diskutiert. Insbesondere der Nutzen der thrombolytischen Therapie ist bislang nicht ausreichend geklärt. Eine aktuelle Metaanalyse aus den USA hat dazu nun Daten hervorgebracht. JAMA 2014; 311: 2414-2421

Die Behandlung einer Lungenembolie mittels Thrombolyse geht insgesamt mit einer verminderten Sterblichkeit einher, führt aber zu einem erhöhten Blutungsrisiko. Zu diesem Ergebnis kommen S. Chatterjee und Kollegen, die insgesamt 16, bis April 2014 veröffentlichte Arbeiten mit 2115 Patienten in ihre Metaanalyse aufgenommen hatten. Die Wissenschaftler berücksichtigten dabei Studien mit randomisiertem, kontrolliertem Design, bei denen die Intervention in einer thrombolytischen Therapie bestand, die mit der Gabe von Heparin, Vitamin-KAntagonisten oder Fondaparinux verglichen wurde.

Für die Metaanalyse wurden primär die Gesamtsterblichkeit und Blutungsrisiken beurteilt, darüber hinaus wurde als sekundäres Outcome die Häufigkeit von Re-

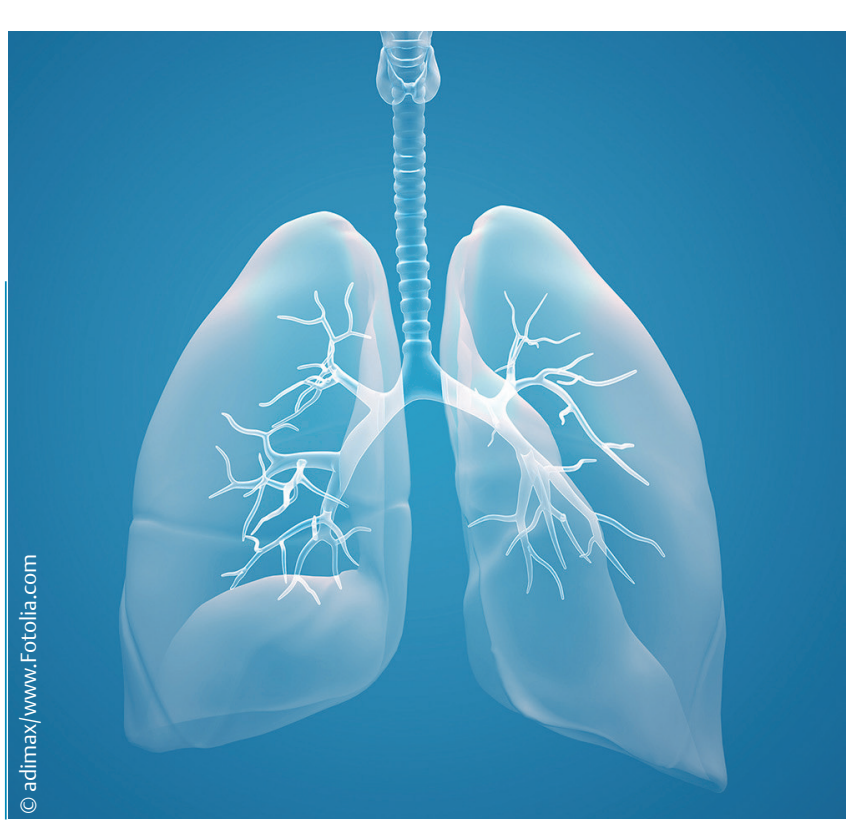

zidiven der Lungenembolie und von intrakraniellen Blutungen analysiert. Anhand des Risikos wurden 3 Patientengruppen unterschieden:

- geringes Risiko - hämodynamisch stabile Patienten ohne Hinweis auf rechtsventrikuläre Funktionsstörung $(\mathrm{n}=210 ; 9,93 \%)$

- mittelgradiges Risiko - hämodynamisch stabile Patienten mit objektiv nachgewiesener (echokardiografisch oder mittels kardialer Biomarker) rechtsventrikulärer Funktionseinschränkung ( $\mathrm{n}=1499 ; 70,87 \%$ )

- hämodynamisch instabile Patienten mit systolischen Blutdruckwerten $<90 \mathrm{mmHg}(\mathrm{n}=31 ; 1,47 \%)$

Knapp ein Fünftel der Patienten konnte wegen unzureichender Angaben nicht klassifiziert werden.

In der vorliegenden Studie wurde von jährlich 100000 Lungenembolie-Fällen in den USA berichtet, davon endeten rund $25 \%$ tödlich. Und auch wer eine Lungenembolie überlebt muss mit erheblichen, gesundheitlichen Einschränkungen rechnen.

\section{Weniger Todesfälle, erhöhte Blutungsgefahr $\nabla$}

Die Auswertung ergab ein insgesamt geringeres Sterblichkeitsrisiko mit thrombolytischer Therapie von 2,17\% (23 von 1061 behandelten Patienten) gegenüber 3,89\% (41 von 1054 Patienten) nach einer mittleren Nachbeobachtungszeit von 81,7 Tagen. Daraus errechnete sich eine Odds Ratio(OR) von 0,53 und eine Number Needed to Treat(NNT) von 59. Dem stand eine Rate schwerer Blutungsereignisse (definiert in den jeweiligen Einzelstudien) von 9,24\% (98 von 1061 Patienten) im Vergleich zu 3,4\% (36 von 1054 Patienten) unter konventioneller Antikoagulanzien-Therapie gegenüber; entsprechend einer $\mathrm{OR}$ von 2,73 und einer Number Needed to Harm (NNH) von 18.

Außerdem ging die Thrombolyse mit einem höheren Risiko für intrakranielle Blutungen einher (OR 4,63; NNH: 78) und einem geringeren Risiko eines Lungenembolie-Rezidivs (OR 0,40; NNT: 54). Eine Subgruppenanalyse ergab weithin, dass die Minderung des Sterblichkeitsrisikos bei älteren Patienten (>65 Jahre) geringer ausfiel als bei jüngeren, das Blutungsrisiko war bei ihnen dagegen deutlich höher. Betrachtete man ausschließlich Patienten mit mäßig ausgeprägtem Risiko, so fand sich bei einer Thrombolyse im Hinblick auf die Sterblichkeit eine OR von 0,48 und für schwere Blutungen von 3,19, jeweils im Vergleich zu einer Antikoagulation.

\section{Fazit}

Die thrombolytische Therapie einer Lungenembolie kann zwar die Zahl der Todesfälle vermindern, ist aber mit der Gefahr schwerer Blutungen verbunden, vor allem bei älteren Patienten. Nach Meinung der Autoren kann die Thrombolyse vor allem bei jüngeren, sorgfältig ausgewählten Patienten einen echten Vorteil bieten; das müssten aber Studien mit längerer Nachbeobachtungszeit weiter klären. Außerdem war in der vorliegenden Metaanalyse nur eine Studie mit kathetergestützter lokaler Thrombolyse enthalten - auch das ist ein Ansatz, der das Blutungsrisiko minimieren könnte und der weiter untersucht werden sollte, so die Autoren.

Dr. Elke Ruchalla, Trossingen 